\title{
A T1OWA Fuzzy Linguistic Aggregation Methodology for Searching Feature-based Opinions
}

\author{
Jesus Serrano-Guerrero $^{\mathrm{a}, *}$, Francisco Chiclana ${ }^{\mathrm{b}, \mathrm{c}}$, Jose A. Olivas $^{\mathrm{a}}$, Francisco P. Romero ${ }^{\mathrm{a}}$, Elmina Homapour ${ }^{\mathrm{b}}$ \\ ${ }^{a}$ Department of Technologies and Information Systems, University of Castilla-La Mancha, Ciudad Real, Spain \\ ${ }^{b}$ Institute of Artificial Intelligence (IAI), School of Computer Science and Informatics, De Montfort University, Leicester, UK \\ ${ }^{c}$ Andalusian Research Institute on Data Science and Computational Intelligence (DaSCI), University of Granada, Granada, \\ Spain
}

\begin{abstract}
Online services such as Amazon, Tripadvisor, Ebay, etc., allow users to express sentiments about different products or services. Not only that, in some cases it is also possible to express sentiments about the different features characterizing those products or services. Most users express sentiments about individual features by using numerical values, which sometimes do not allow users to reflect properly what they are meaning and therefore they are misleading. To overcome this key issue and make users' opinions in online services more comprehensive, a new methodology for representing sentiments using linguistic term sets instead of numerical values is presented. In addition, this methodology will allow to implement importance degrees on the different features characterizing users' opinions. From both sentiments and importance of the features, the most important opinions for each user is derived via an aggregation step based on the Type-1 Ordered Weighted Averaging (T1OWA) operator, which is able to aggregate the corresponding fuzzy set representations of linguistic terms. Furthermore, the final output of the T1OWA based-search process can easily be interpreted by users because it is always of the same type (fuzzy) and defined in the same domain of the original fuzzy linguistic labels. A case study is presented where the T1OWA operator methodology is used to assess different opinions according to different user profiles.
\end{abstract}

Keywords: Sentiment Analysis, Feature opinion aggregation, Linguistic Model, T1OWA aggregation operator

\section{Introduction}

Retrieving information containing opinions towards different products is recently becoming a real need of many users on the Internet. Many notable online platforms like Amazon ${ }^{1}$ or Ebay ${ }^{2}$ allow users to express sentiments about their products and, as Tripadvisor ${ }^{3}$ does, about the features of those products. However, 5 when a user is looking for some information about a specific product, usually the only filtering mechanism offered is the simple selection of those opinions sharing the same overall rating. Therefore, in practice, users who are only interested in specific features of a product cannot find good or bad products regarding those specific features. For example, when a user is searching for a hotel, most websites available on the Internet may rank hotels according to the overall rating given by past clients. This mechanism is quite rigid because it does not

\footnotetext{
*jesus.serrano@uclm.es

Email addresses: jesus.serrano@uclm.es (Jesus Serrano-Guerrero), chiclana@dmu.ac.uk; inv.chiclana@ugr.es (Francisco Chiclana), joseangel.olivas@uclm.es (Jose A. Olivas), franciscop.romero@uclm.es (Francisco P. Romero), elmina.homapour@dmu.ac.uk (Elmina Homapour)

${ }^{1}$ https://www.amazon.com

${ }^{2}$ https://www.ebay.com

${ }^{3}$ https://www.tripadvisor.com
} 
allow a user to rank hotels according to his/her preferences, which may be expressed via individual particular features describing the hotel (see Figure 1) such as: cleanliness, service, value, etc. In addition, the importance of each of these features may be different for each user, therefore, a mechanism to express personalized user's needs is required.

In addition, some of these online platforms (websites) allow their users to rate the final service/product in a different way to the individual features describing such service/product. Indeed, a user can write that a hotel has a bad rating for individual features like cleanliness, service, value, etc., but provides a positive overall rating, which could lead to the provision by users of fake or incoherent opinions. Moreover, the mechanism for rating products or services on these platforms is usually numeric valued based, and many users may not feel comfortable with when scoring features. Many users may find quite different the rating of the battery of a mobile phone with a 2 stars than with the linguistic expression "really bad". Linguistic assessments are more intuitive, informative and understandable than numeric ones. For instance, looking at the TripAdvisor entry opinion on a hotel reproduced below in Fig. 1, one may conclude that the user is not reflecting adequately with stars with the elaborated description provided. The adjective "pretty standard" does not appear to match high values of 4 and 5 stars.

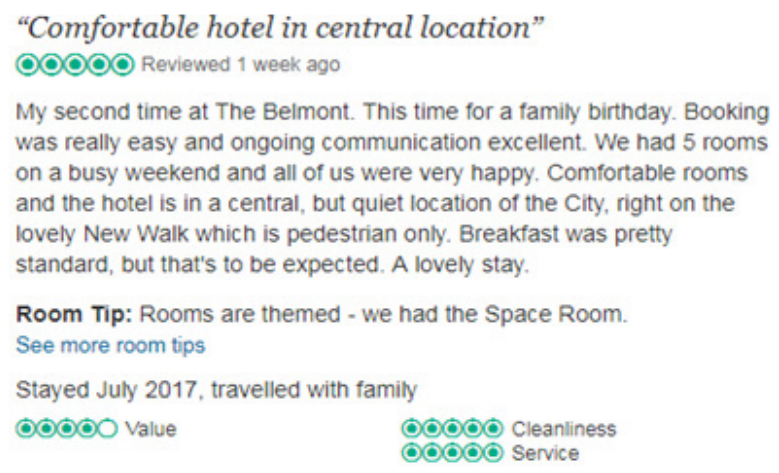

Figure 1: Opinion from TripAdvisor

This paper proposes a new methodology that allows users to interact in a more intuitive way while expressing their opinions and that is also capable of filtering the most relevant opinions according to their needs. This mechanism is based on the modelling of needs and sentiments via fuzzy variables, as a tool for representing linguistic information. The proposal also presents a mechanism to aggregate the sentiments of all the available features of a product/service to automatically generate its final overall score, releasing the user from doing it, so that it more reliable and consistent with respect to the individual sentiments expressed about each of the assessed features. To the best of our knowledge, little work can be found on mechanisms for personalized opinions search. As a consequence, the main contributions of this article are:

(i) Design of a fuzzy based mechanism to compute preferences and sentiments expression via linguistic values instead of numerical values;

(ii) Implementation of a fuzzy based aggregation mechanism to compute overall scores depending on individual facet linguistic sentiments that adapts to the user preferences; and

(iii) Put forward real scenarios in which the two former contributions may work successfully.

The remainder of this work is organized as follows: Section 2 briefly reviews the most relevant literature on Sentiment Analysis and Aggregation research for the present paper. Section 3 presents examples of different 
opinions that motive the proposal put forward in this article, which is fully detailed in section 4 . Section 5 shows some examples applying the methodology previously described. Finally, section 6 points out some conclusions and future research work.

\section{State of the Art}

\subsection{Sentiment Analysis}

Interest in Sentiment analysis, also known as Opinion Mining, has been growing steadily in the last years, mainly because of its great number of applications and the scientific challenges it poses. Its main purpose is the analysis of people's sentiments (opinions, attitudes, emotions, etc.) regarding topics, products, individuals, organizations, and services. Sentiment Analysis is closely related to other fields such as Artificial Intelligence (AI), Information Retrieval (IR) an Natural Language Processing (NLP). Indeed, NLP is very important for Sentiment Analysis because many aspects, such as Word Sense Disambiguation [1] among others, can determine whether it is possible to detect whether opinions are available or not. One of the main aspects that NLP has to deal with is the different levels of analysis. This is key because different types of tasks and texts are to be tackled [2] depending on whether the target of the study is a whole text or document, one or several linked sentences, or one or several entities or features of those entities. Hence, there is three distinct levels of analysis in Sentiment Analysis: (i) the document level, (ii) the sentence level and (iii) the entity/feature level.

At the document level, a document is considered an opinion on an entity or on a feature of an entity, and the task at this level is called document-level sentiment classification [3, 4, 5, 6, 7, 8, 9]. Nevertheless, when a document contains several sentences related to different features or entities, then the sentence level is more appropriate, and the task at this level is called subjectivity classification to distinguish sentences expressing factual information from those sentences expressing subjective views and opinions [10, 11, 12, 13, 14]. Finally, if more precise information is required, then the entity/feature level is applied, which is the finest-grained level because it targets whether the opinion holder expresses a positive or negative opinion. This latter level is possibly the most complex as well because it is necessary to extract with high precision among other: dates or time spans, the different features and entities to be opinionated along with their relationships, the opinion holders and their characteristics. Thus, this level tasks are closely related to feature-based opinion mining and opinion summarization [15, 16, 17, 18. The inclusion of other factors different to text such as users influence and trust [19, 20] would make possible to classify sentiments from communities extracted from social networks [21], or such as video and/or audio features would lead to multimodal sentiment aggregations [10].

Sentiment Analysis is a very broad field and it encompasses, among other, the following different subareas: sentiment classification, subjectivity classification, opinion retrieval, sarcasm and irony, emotions [22, 23, 24]. In particular, this work is closely related to opinion summarization, also known as opinion aggregation, and it especially focuses on extracting the main features of an entity shared within a single or a multi-document and the sentiments with regard to them [25]. Single-document summarization consists in analyzing internal facts present within a document, for example, changes in the sentiment orientation throughout the document or links between the different entities/features found in the document by showing those pieces of texts in the document that better describe them. On the other hand, in multi-document summarization the different sentences that express sentiments related to the detected entities or features are grouped and/or ordered. The final summary might be presented as a graphic or a text showing the main features/entities and quantifying the sentiment with 
regard to each one by, for example, aggregating intensities of sentiments or counting the number of positive or negative sentences [26, 27]. According to [28, in opinion summarization, the aggregation of the different elements of an opinion requires to carry out several phases as depicted in figure 2

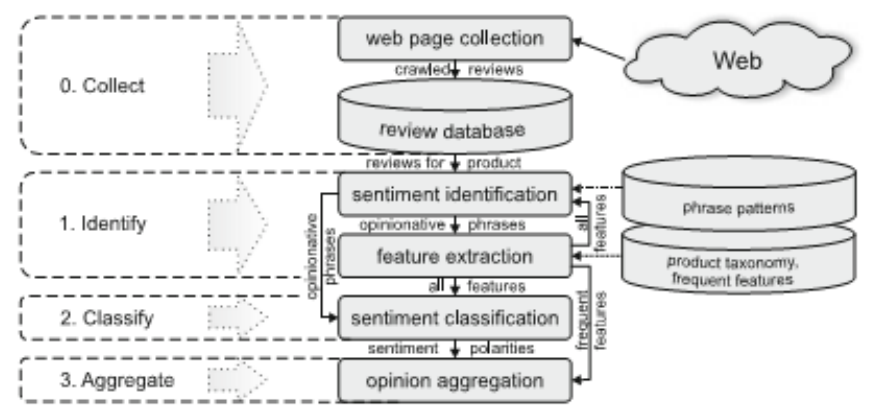

Figure 2: Architecture of product review aggregation [28]

In the literature, it is possible to find approaches for aggregating sentences to obtain the general sentiment for a document [29]; which is not the case for feature-level aggregation, except for the prediction of individual feature ratings [30, 31]. Nevertheless, "most methods aggregate sentiment by simply averaging or taking a majority vote" 32, i.e. most existent approaches are simple numerical but not linguistic based approaches [33]. For example, in [34, the authors proposed an ontology-based framework that ranks/summarizes the main features of different products that is based on the aggregation of the detected user's preferences. On the other hand, in 35 an ontology-based strategy is presented where the importance weights of the different features depend on the levels of an ontology developed by using Conceptnet [36], which are subsequently used to aggregate the sentiments on each feature. In [37, it was proposed the aggregation of a semantic score and a credibility score as main aspects to obtain a final numeric value in determining the sentiment towards some products, while in 38, several numerical approaches, based on the mean or a weighted mean operator, were propose to fuse the polarities from several features from Amazon's products. On the other hand, when aggregating several opinions, it may also necessary to take into account variables such as time. Sentiments can vary over time and, therefore, sentiment aggregation might lead to contradictory results depending on the dates when the aggregated opinions were written, which has resulted in a new area in Sentiment Analysis known as Sentiment Diversity of Sentiment Contradiction [39].

\subsection{The Type-1 OWA operator}

An Ordered Weighted Averaging (OWA) operator [40] of dimension $n$ is a mapping $F: R^{n} \rightarrow R$ with an associated weighting vector $W=\left[w_{1}, w_{2}, \ldots, w_{n}\right]$, subject to the constraints $w_{j} \in[0,1]$ and $\sum_{j=1}^{n} w_{j}=1$, that maps an input vector $\left(a_{1}, a_{2}, \ldots, a_{n}\right)$ with an output scalar value $F\left(a_{1}, a_{2}, \ldots, a_{n}\right)=\sum_{j=1}^{n} w_{j} b_{i}$, being $b_{j}$ the $j$ th-largest input vector component.

Inspired by the OWA operator, and the fuzzy extension principle, the type-1 OWA (T1OWA) operator was defined in [41] to allow the fusion of uncertain information with uncertain weights expressed linguistically and represented as (type-1) fuzzy sets (T1FSs). A T1FS $A$ on a larger set $X$, normally referred to as universe of discourse, is characterized by a numeric membership function $\mu_{A}: X \rightarrow[0,1]$ that associates to an element $x \in X$ its membership to $A, \mu_{A}(x) \in[0,1]$. Denoting the set of all T1FSs on $X$ by $F(X)$ and $U=[0,1]$, the mathematical formulation of the T1OWA operator is given below. 
Definition 1. "Given n linguistic weights $\left\{W_{i}\right\}_{i=1}^{n}$ in the form of T1FSs on $U$, an associated T1OWA operator of dimension $n$ is a mapping that aggregates type-1 fuzzy sets $\left\{A_{i}\right\}_{i=1}^{n}$ on $X$ in the following way:

$$
\begin{gathered}
\Phi: F(X) \times \cdots \times F(X) \quad \longrightarrow F(X) \\
\left(A_{1}, \cdots, A_{n}\right) \quad \mapsto G \\
\mu_{G}(y)=\sup _{\sum_{k=1}^{n} \bar{w}_{i} a_{\sigma(i)}=y}\left(\mu_{W_{1}}\left(w_{1}\right) * \cdots * \mu_{W_{n}}\left(w_{n}\right) * \mu_{A_{1}}\left(a_{1}\right) * \cdots * \mu_{A_{n}}\left(a_{n}\right)\right)
\end{gathered}
$$

where $*$ is a $t$-norm operator, $\bar{w}_{i}=\frac{w_{i}}{\sum_{i=1}^{n} w_{i}}$, and $\sigma:\{1, \cdots, n\} \rightarrow\{1, \cdots, n\}$ a permutation function such that $a_{\sigma(i)}$ is the $i$-th largest element in the set $\left\{a_{1}, \cdots, a_{n}\right\} . "$

An direct approach to perform expression (1) was developed in [42, which due to its high computational complexity was optimised with a faster $\alpha$-level implementation in [4]. The T1OWA aggregation operator is especially interesting for implementing the cardinal fuzzy approach to linguistic information representation, which is more "general, flexible, and appropriate to capture uncertainty" than the ordinal approach [4].

\section{Motivation}

Different behaviors may be detected by analyzing users' opinions on the Internet. In most cases, the use of just numerical values to score opinions is not the most suitable mechanism not it is appropriate to characterize user profiles, which would require of additional variables. In fact, these additional variables would help users to find the most adequate opinions with regard to their needs. The following examples illustrates the points just raised.

\subsection{Type of users}

Any classification method depends on the different aspects that are of interest. These are some which make understandable the need of having a mechanism for representing opinions like the proposed here.

\subsubsection{Coherent}

It is not uncommon to find users' opinion and their ratings to not match, which makes the system presenting them to be perceived as inconsistent and tricking the reader with information that is not trustworthy.

Coherent user. From the opinion shown in Fig. 3 it is noted that the user utilizes different adjectives that fit with a maximum rating ("great", "excellent") regarding the hotel in general and the services it offers in particular, while the rest of descriptors used ("easy", "friendly", "good") regarding localization, staff, food and facilities, respectively, are just below the maximum rating. Nonetheless, in this example the user is giving the maximum score value to the hotel, which might lead potential readers to believe that the hotel is "perfect" when actually it is not according to the details referring to localization, staff, food and facilities. Therefore, users, searching on the Internet for excellent hotels, who take into account the overall rating as their main criterion, would very likely be returned many hotels by this system that will not meet their expectations. 


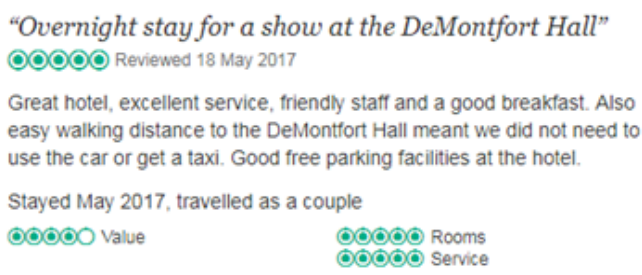

Figure 3: Coherent opinion

Incoherent user. Looking at the following entry shown in Fig. 4), it is clear that textual comments and ratings do not match. Adjectives like "good" (for night sleep) does not reflect the maximum scoring of 5 received by the category "Sleep Quality", being the adjectives "perfect" a better one as used for describing the category "Localization". Again, "good" was used for room size, which leaves room for a "better" size, it was "tidy" and

"clean" but not "superb'. Therefore, the user's opinion seems to be incoherent with respect to the rating values used, which leads to argue that the mechanism used to express these ratings is not intuitive and/or expressive enough. This suggests the need to develop a different mechanism to improve and facilitate the provision and representation of descriptive opinions by users.

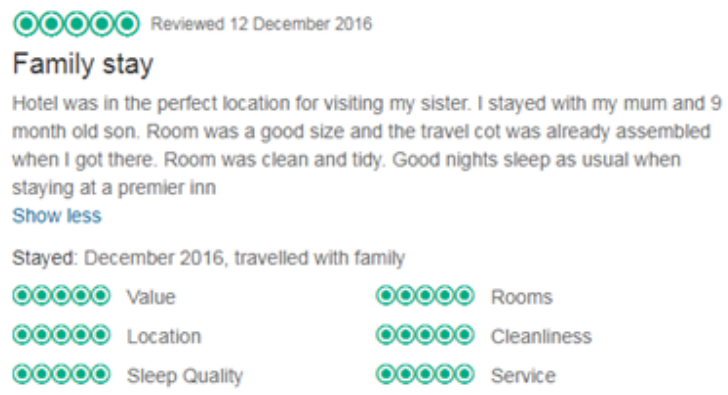

Figure 4: Misleading opinion

\subsubsection{Haters and lovers}

Sometimes it is necessary to filter many opinions because they may not reflect the reality. For example, Fig. 5 illustrates a negative experience of a user due to a misunderstanding while booking a hotel room. The additional fact of the pool being out of service at the time of that user's stay is an additional factor in this user's experience being negative. As a consequence, all features were assessed with the minimum rating without any evidence to back up them all. It appears obvious that this opinion was given in retaliation.

Contrary to the above user's opinion, Fig. 6 shows the opinion of a user, who states that everything was perfect, that does not match another user's opinion on the same hotel given on the exact same day, reproduced in Fig. 7), which seem to be a fairer and more balanced with a variety of ratings (positives and negatives) used for the set of individual features and a overall rating of the hotel being located just below their median value.

\subsubsection{Mood}

It is also necessary to take into account the moment in which an opinion is expressed. It is better not to express opinions when in a bad mood because the it could lead to an overall rating for the hotel worse than the real deserved one. For example, Fig. 8 shown a review of a user rating all features of the hotel with maximum 


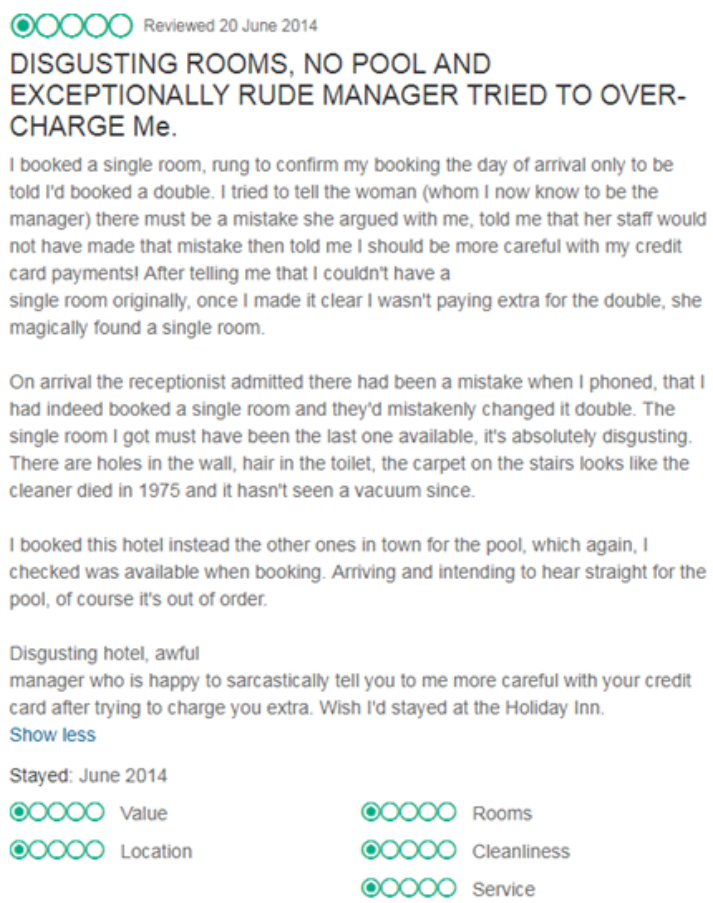

Figure 5: Hater

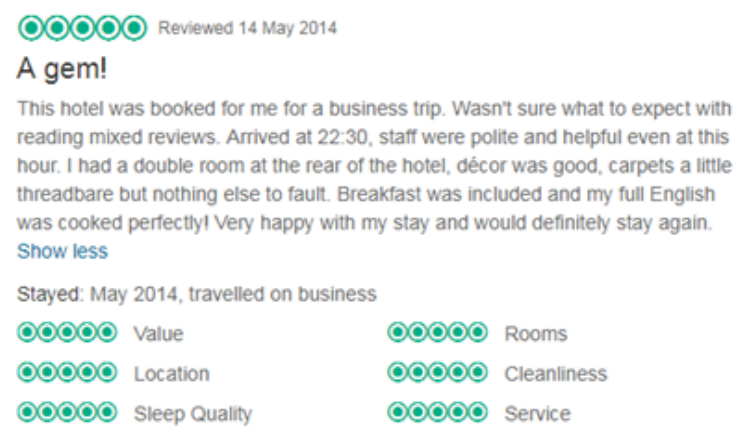

Figure 6: Lover

ratings but one, whose rating is used for the overall rating of the hotel. This is an example of an unbalance used of the individual feature ratings to derive the overall rating of the hotel.

A more balance approach to derive the overall rating from the individual feature ratings is exemplified in the entry shown in Fig. 9, where a more wide use of different ratings used for the set of individual features, being a majority in the negative scale of ratings, which was subsequently used for the overall rating of the hotel.

The examples examined provide evidence of the difficulty users may experience in finding coherent and/or balanced, i.e. reliable, information. Indeed, when a user is looking for good hotels, the interface shown in figure 10 would not present the opinion presented Fig. 8 because its overall rating does not belong to the "Good" or "Excellent" categories of such interface although the written statement refers to such a type of hotel. It can be concluded that there is a need for a mechanism to model and deal with opinions similar to the ones exemplified above and that is able to produce coherent and balance information based on the individual feature ratings. 


\title{
OOOOO Reviewed 14 May 2014
}

\section{not so good as it used to be}

We have stayed here 3 times but never again, the rooms were very clean but the hot water tap in the bathroom would not deliver any hot water.when we went down to reception the receptionist was outside smoking, she did change our room without any fuss. I understood breakfast was included but apparently not as there is another charge on my bank account. the breakfast for me was the worst I have had in an hotel. coffee was cold, sausage of very poor quality, mushrooms were not even cooked but chopped up cold on the plate tomato was not grilled and black pudding was cooked so much it was rock hard. there was no mention of the restaurant being closed on Friday and Saturday nights. drinks at the bar they wanted us to pay with a card as they had no change in the till ,beer was rubbish and my coke served in a dirty glass, I think the love has gone out of this hotel. swimming pool was empty and the room was unkempt with autumn leaves still on the floor. back exit door to the car park needs to be replaced it looks like someone had tried to kick it in. very disappointed this time and will not return Show less

Stayed: May 2014, travelled as a couple

\begin{tabular}{|c|c|c|c|}
\hline ○)000 & Value & ○ంO০O & Rooms \\
\hline ○ంலం & Location & ○ం০ం0 & Cleanliness \\
\hline$D O O O$ & Sleep Quality & $\infty$ & Service \\
\hline
\end{tabular}

Figure 7: A more balanced opinion

\author{
OOOOO Reviewed 8 March 2014 \\ Mixed bag! \\ PLEASE read whole review.
}

I booked into an executive room for comfort, but also requested ground floor. I was given a HUGE family room rather than a designated executive room (room 31) This is a great room for families as it has two extra large single beds as well as the main one.

The bathroom was cavernous with a bidet and a bath. The shower was rubbish. fixed head and the hot water in the bathroom is only tepid.

The room is very cold and the radiators just lukewarm. I was given a fan heater and this worked well.

The problem it appears is that from a heating point of view they have knocked 2 rooms into one but not installed adequate radiators.

The hotel was taken over last year, after it had been let to run down by the previous management company. The present owners are trying hard to update it. Unfortunately the leisure centre (including the pool) are not open. The pool will not re open.

The restaurant is not very appealing and the prices are not cheap. The general decoration is dingy in the communal area.

If you are disabled there is no lift so you need to ask for a downstairs room, but I don't think there is an adapted room.

On the plus side the staff are the most helpful I have met in a hotel for a long ime. They really tried hard to rectify the problems and I cannot fault any of them.

This hotel is in a perfect location in Blaby as it is near bus routes and just a few minutes stroll to a good variety of shops. There is a massive car park as well. Reception is at the side of the building.

If you have to visit Blaby then this hotel is very adequate and the prices were good. I would recommend it because the staff are great and in spite of being very dingy it is clean and that is why I said read the whole review.

Show less

Room Tip: I had room 31 on the ground soor. It was very quiet and very clean. View onto coen car was.

Stayed: March 2014, travelled on business
oOOO Value
OOOOO Rooms
OOOO Location
○○OO Cleanliness
OOOO Sleep Quality
○o000 Service

Figure 8: Opinion given in a bad mood 


\section{OOOOO Reviewed 7 April 2014}

Never again

I stayed here between Fri-Sun on business travel. The blaby seems to be a hotel that has struck on hard times, and for the owners in this regard I feel bad. The staff seemed pleasant and there was always somebody around reception. But the hotel itself was awful, and reminiscent of a hostel or budget lodgings. Cleanliness was spot on and I cannot fault it, the team deserve a pat on the back. But the

ammenieties whilst at the hotel were somewhat non-existent and I was spoken to as if $i$ was stupid to expect otherwise. The restaurant was closed the whole time, and I wasnt made aware of this until the first evening after a hard day working and a hungry belly. I was simply told the restaurant was closed, I wasnt given directions to a nearby restaurant etc. Waitrose for a sandwhich is was then!

Secondly, breakfast is usually served 7 am in hotels especially for business

travellers. I went down for breakfast at 7 am, only to be told it won't be until 8 am over the course of my stay. I started work at $8 \mathrm{am}$, so no breakfast for mel

On the website it was advertised that there was a pool. This was half drained and closed off during my stay.

I was moved to another room, quite kindly, on my second night due to a noisy room the first. The room was spotless, but the bathroom floor was heavily cracked an lifting up after a possible leak. The room shouldnt have been given.

It seems that this hotel has closed its restaurant, pool and have limited cleaning provisions (only some rooms were being given due to cleaning staff not in everyday) to keep afloat. For this I feel bad for the owners, but you can't charge for a service that is only marginally B\&B.

A very very dark, dated hotel that I couldn't wait to leave. I left feeling depressed and wanting a decent meal and to get homel

Show less

Stayed: April 2014, travelled on business

OOOOO Value

OOOOO Rooms

○OOO Location

○OOO Cleanliness

@OOO Sleep Quality

o0000 Service

Figure 9: A realistic opinion

\section{Overview}

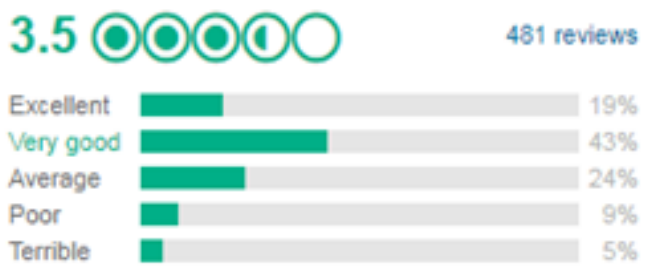

Figure 10: Interface to select opinions 


\section{Methodology}

Each product/service to be opinionated is represented by a finite set of features, $\left\{F_{1}, F_{2}, F_{3}, \ldots, F_{N}\right\}$. These features will be used to represent users' profile and opinions as described below. The proposed methodology framework is depicted in Fig. 11 .

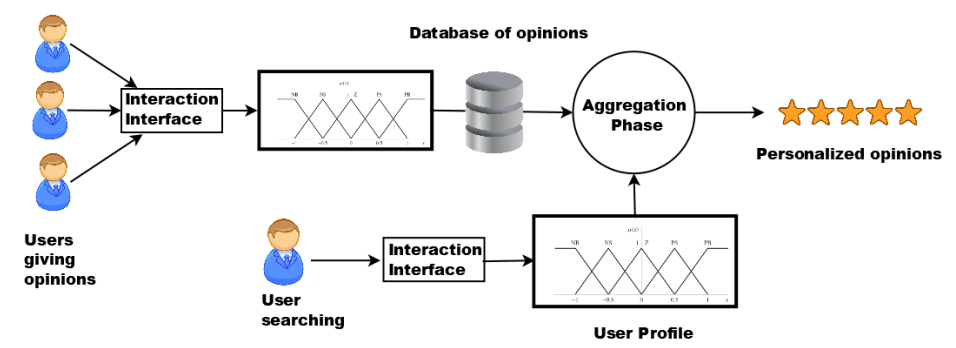

Figure 11: General diagram

\subsection{Users'opinion (UO)}

Users are allowed to post comments on their experiences and, as a result, a (large) database of opinions will be available to other users looking for information on products/services. Thus, in practice, based on their own experiences, users express their opinion regarding the different features of a given product or service.

Users' opinions on each feature will be summarised by linguistic labels belonging to a set $S_{o}=\left\{S_{o 1}, \ldots, S_{\text {om }}\right\}$, which for computation purposes will will be represented as Triangular Fuzzy Numbers (TFNs) with corresponding membership function

$$
\mu(x)= \begin{cases}0 & 0 \leq x<a \\ \frac{x-a}{b-a} & a<x \leq b \\ \frac{c-x}{c-b} & b \leq x<c \\ 0 & c<x \leq 1\end{cases}
$$

Thus, a TFN is simply represented by a 3 parameter-tuple $(a, b, c)$, with $a$ and $c$ being its lower and upper bounds, respectively, and $b$ its modal value. Symmetrical TFNs verify that $b=(a+c) / 2$, which is also the centroid of the TFN. Depending on the "discriminative power" to use and implement and the parameters chosen, a balanced or unbalanced set $S_{o}$ of linguistic labels can be obtained. A balanced set of 5 labels and an unbalanced set of 7 labels are shown in Fig. 12 and Fig. 13, respectively, with the first one having a lower discriminative power than the second one.

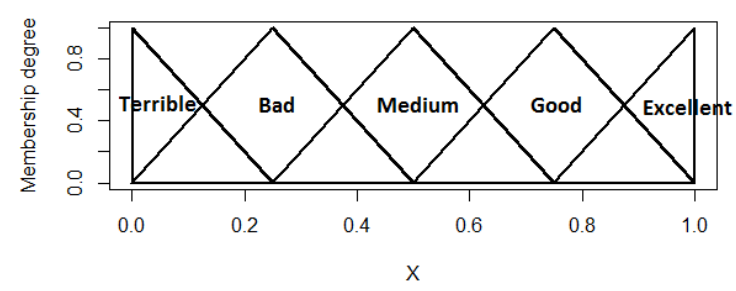

Figure 12: Balanced set of 5 linguistic labels

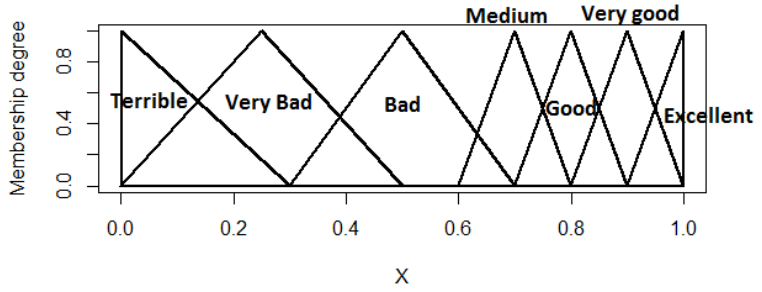

Figure 13: Unbalanced set of 7 linguistic labels

A user opinion will therefore be represented mathematically by a set of $N$ linguistic labels from the set $S_{o}$, $U O=\left\{U O_{1}, \ldots, U O_{N}\right\}$. 


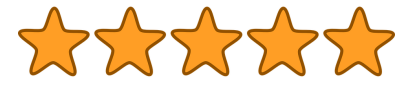

Figure 14: 5-star rating

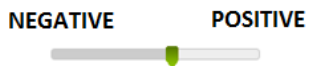

Figure 15: Opinion bar

\subsubsection{Interaction interface}

Once the features and the set of linguistic labels $S_{o}$ have been defined, the user has to able to express his/her opinions about items. To do so, a user interface is necessary. Different platform offer different ways of doing this, although it is usually done using a scale graphical represented to make it intuitive to use, like the graphical scale based on a set of stars shown in Fig. 14 representing the ordinal scale from 1 to 5 . An alternative option is the use of an opinion bar 32 as shown in Fig. 15 , which allows for a continuum evaluation in a closed interval, which could be set to be $[0,1]$.

The fuzzy set based approach uses linguistic labels rather than numbers. Their relationship would be as follows. In the first numerical scale, 3 stars may represent a "medium" opinion while 5 stars an "excellent" opinion. In the second case, the membership value of a number in $[0,1]$ selected with an opinion bar is computed for each fuzzy linguistic labels of the predefined label sets, and those labels with a positive membership value (activation level) will be activated.

As a consequence, the proposal here presented can deal with schemes containing different numbers of labels, it is not necessarily a direct correlation between the number of labels and the starts used to rate a product/aspect.

\subsection{Users' profile (UP)}

An important issue to address is the definition of users' profile. When users are looking for some information, they would like to have information specially adapted to their needs. For that reason, it is necessary to measure the importance of each feature according to each user, because the system needs to know exactly what the user is interested in.

Therefore, users must be able to express their preferences about the different features of a product or service with regards to their importance. Similarly to the case of users' opinions mentioned before, linguistic labels belonging to a set $S_{u}=\left\{S_{u 1}, \ldots, S_{u m}\right\}$, represented as TFNs, will be used for such task. Similarly, depending on the "discriminative power" to use and implement, the granularity or "discriminative power" level will be selected and the corresponding parameters of the TFNs will be chosen in order to reflect whether $S_{u}$ is a balanced or unbalanced set of linguistic labels. Fig. 16. shows a possible balanced set of five labels for users to specify their importance for each feature of a product/service. The set of linguistic labels $S_{o}$ used to assess the users' opinion is not necessarily equal to the set used for the users' profile $S_{u}$.

A user profile will therefore be represented mathematically by a set of $N$ linguistic labels from the set $S_{u}$, $U P=\left\{U P_{1}, \ldots, U P_{N}\right\}$.

\subsection{Aggregation phase}

Once the users' opinions and the users' profiles are known, the T1OWA operator is applied to aggregate the set of fuzzy labels representing the sentiments of all features according to the user's opinion $U O$, using as weights the set of fuzzy labels representing the corresponding user's profile $U P$. The aggregated output will be a fuzzy set representing how suitable overall the product/service would be for the user. 


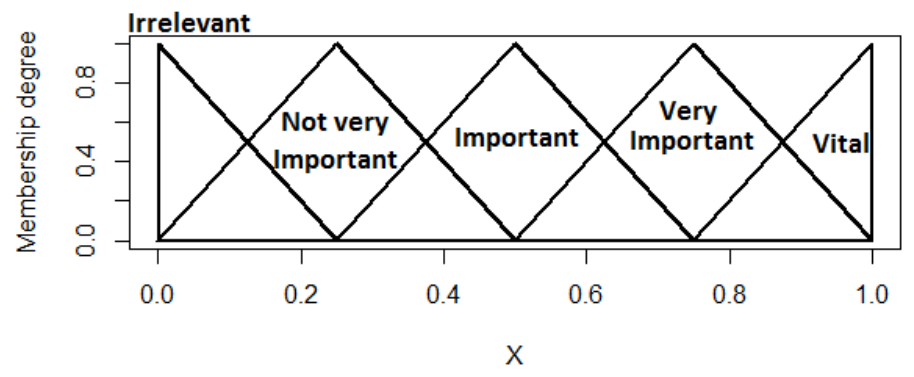

Figure 16: Importance scheme

The comparison of the T1OWA outputs might be necessary to be implemented to allow users looking for a product/service to make a final choice. It is well known that the set of fuzzy sets (numbers) is not totally ordered [45, thus an ordering approach on the use of a metric, such as the Hamming or Euclidean distances [46, 47, 48, via the application of the extension principle will not solve this issue in the fuzzy case. In the fuzzy case, the alternative approach to rank fuzzy numbers consists in converting the fuzzy sets into a representative crisp value, and perform the comparison on them [49].

Two defuzzification methods widely used in fuzzy set theory are: the centre of area method (COA) and the mean of maximum method (MOM). The first one computes the centre of mass of the membership function of the fuzzy set (the centroid), whereas the second one computes the mid-point of the 1-level set of the fuzzy set. The COA method maintains the underlying semantic ranking relation within the set of linguistic labels, it is adopted here, and for a type- 1 fuzzy set $\mathrm{A}$ in a continuous domain $\mathrm{X}$ is calculated as:

$$
U_{C O A}(A)=\frac{\int_{x} x \cdot \mu_{A}(x) d x}{\int_{x} \mu_{A}(x)} .
$$

\subsection{Types of users}

Finally, it is also necessary to distinguish between the different users of a system based on this model. We could look at the system from two different perspectives. On the one hand, the typical client who is looking for some services like booking a hotel, renting a house or buying a phone. On the other hand, a hotel, a state agency or a shop selling phones may be also interested in modeling properly the system to make the most of it. The latter may be more interested in defining a perfect opinion scheme that allows discriminating perfectly between all services offered. For example, a big seller, using a very strict opinion scheme capable of measuring low quality degrees (useless, very very bad, very bad, bad .... ) of its products, could offer different discounts ( $25 \%$ off, $20 \%$ off, $15 \%$ off, $10 \%$ off ... ) in order to sell those low-ranked products in stock. On the other hand, clients are more interested in a perfect representation of the weights characterizing their profile. This way, the system may recommend better opinions to them. This type of user usually start by looking for information about goods and/or services, which might be subsequently followed by their own opinion expression after after using the service or purchasing the product.

\section{Case study}

This section presents some possible applications of the previous proposal. 


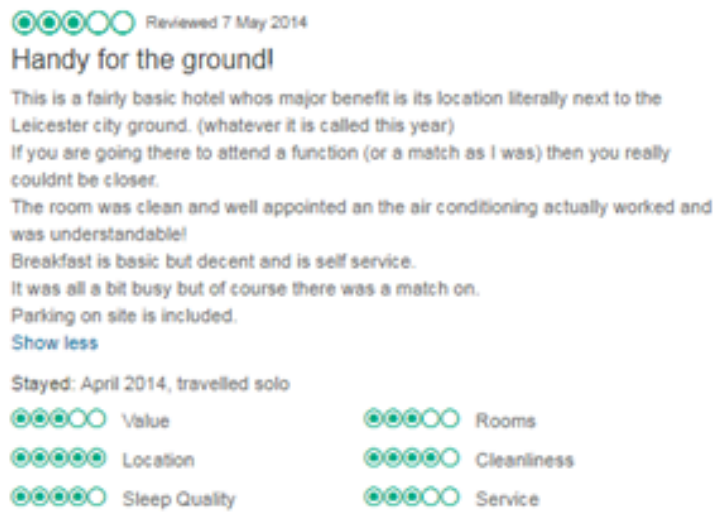

Figure 17: Opinion proposed to test a specific user profile

\subsection{User profile} specification in terms of features' importance.

For a different type of user, like user in town for business matters, location might be less interesting because he/she might take a taxi and other features regarding "laundry" or "restaurant", or "room" might be more interesting if they were to spend more time working at/from the hotel preparing/holding meetings, for example. In these cases, the following $U P$ might be more appropriate: 


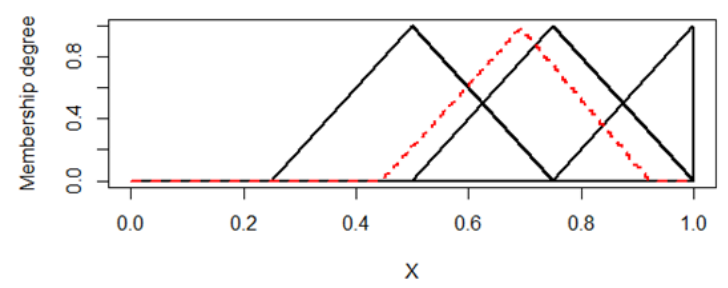

Figure 18: Aggregation regarding a tourist profile

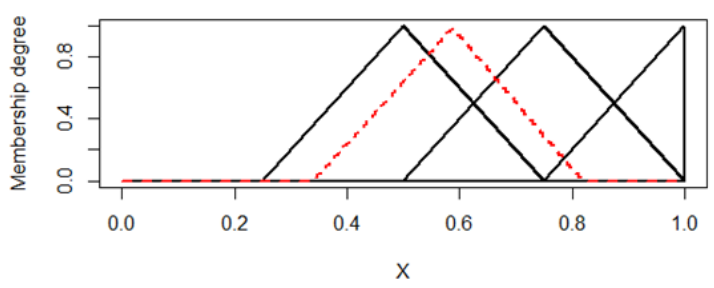

Figure 19: Aggregation regarding a business man profile

- Value $=$ Not important

- Location $=$ Not important

- Sleep Quality = Medium

- Rooms $=$ Vital

- Cleanliness $=$ Medium

- Service $=$ Vital

Applying the proposed model to the previous opinion we get the red dashed line overall output shown in Fig. 19, which indicated that this hotel is less suitable for business users than it is for tourist users. Indeed, in this case the hotel overall assessment is closer to the central fuzzy set with centroid of 0.58 whereas for the tourist user the centroid value was 0.7 .

\subsubsection{Overall rating}

As it can be seen from the different examples above, sometimes the overall rating given by users does not allow telling all hotels apart according to their quality. The strategy of using 5 stars to rate a feature or product/service in general might make the assessment process easier for the user although opinions are not always informative/consistent enough. For that reason, allowing the system to internally compute the overall rating will have the benefit of achieving a less subjective, i.e. less dependent on users, but more consistent with the individual ratings entered by the user. As a consequence, the unbalanced 7-label opinion set of Fig. 13 can be used if we would to make harder for a hotel to receive an overall rating of "Good" and above, and at the same time to allows a better differentiation between "very good" hotels from those that are "excellent" hotels.

Let us consider the opinion shown in Fig. 20 with overall rating being the minimum of the individual feature ratings. To apply the proposed model to this opinion, it is necessary to fuzzify the points/starts ratings of each individual feature. Adopting an optimistic approach, a points/starts rating is mapped to the fuzzy linguistic label with maximum activation level. This opinion is therefore to be treated in the proposed model as follows:

- Value $=\mathrm{Bad}$

- Location $=$ Good

- Sleep Quality = Bad

- Rooms $=$ Medium 


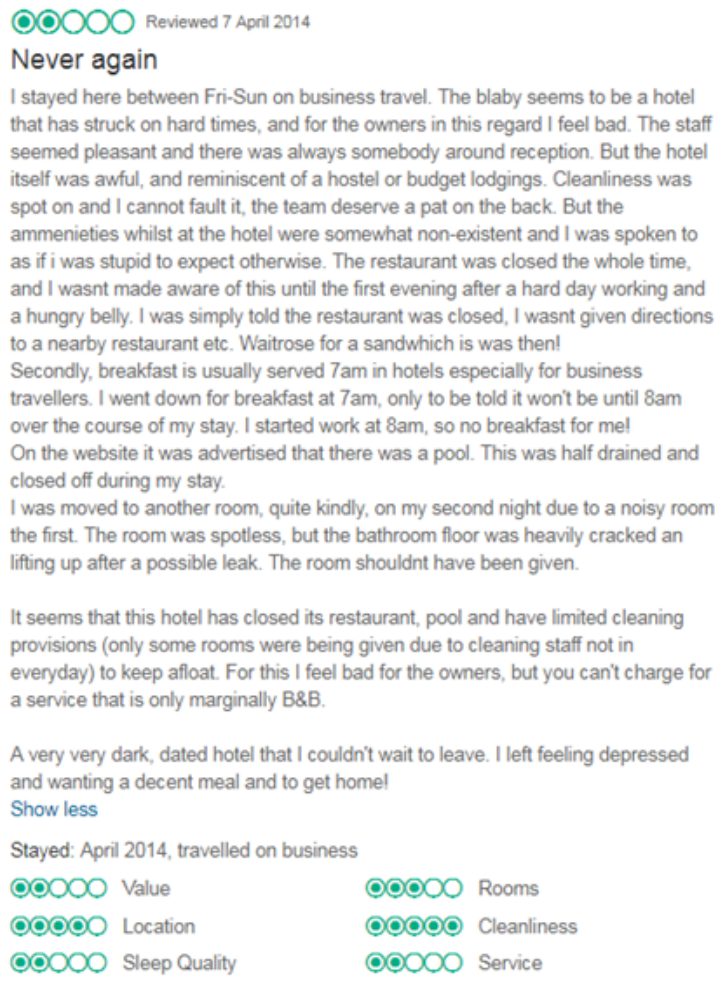

Figure 20: A realistic opinion

- Cleanliness = Excellent

- Service $=$ Bad

Under the assumption of being all features equally important to the user, the proposed model results in an overall rating pretty similar to the label "Bad" as Fig. 21 illustrated with a red dashed line.

If the balanced 5-label opinion set from figure 12 were used instead, then the individual feature ratings would be mapped into the following fuzzy linguistic labels:

- Value = Very low

- Location $=$ Good

- Sleep Quality = Very low

- Rooms = Low

- Cleanliness = Excellent

- Service = Very low

Again, under the assumption of being all features equally important to the user, the proposed model results in the overall rating shown in Fig. 22, which is similar to the overall rating obtained above with the unbalance 7-label opinion set but that in this case it is not represented as "Bad" but as "Medium". Thus, it is clear that the unbalance 7-label opinion set will make harder for a hotel to be categorised good or above than with the balanced 5-label opinion set.

In order to corroborate the above, an additional example with the opinion shown in Fig. 23 is provided.

In this case, balanced 5-label opinion set would lead to the following representation: 


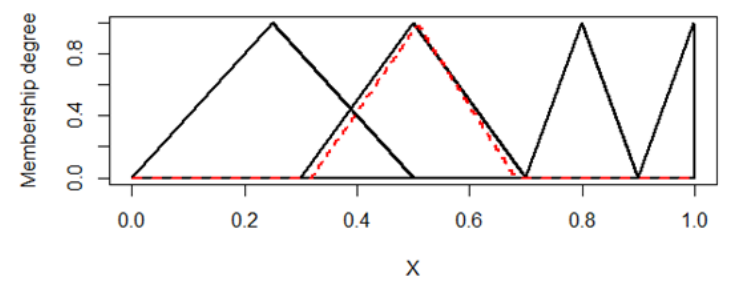

Figure 21: Aggregation with unbalanced 7-labels opinion set

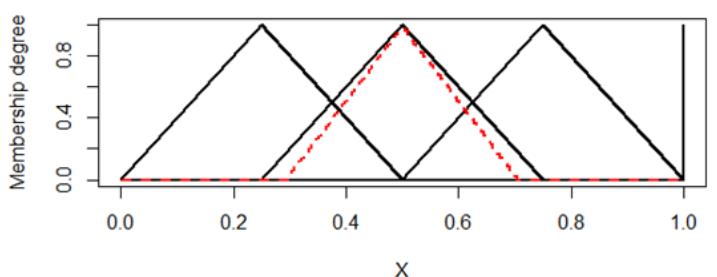

Figure 22: Aggregation with balanced 5-labels opinion set

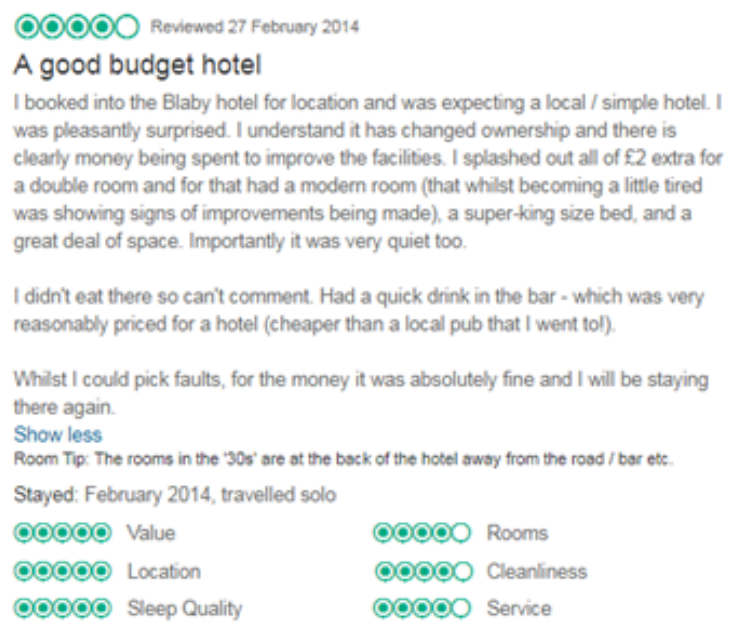

Figure 23: A positive opinion but not excellent

- Value $=$ Excellent

- Location $=$ Excellent

- Sleep Quality = Excellent

- Rooms $=$ Good

- Cleanliness $=$ Good

- Service $=$ Good

Considering all features are equally important to the user, the overall rating obtained using the T1OWA proposed approach is shown in Fig. 24 its centroid being 0.833 and therefore closer to the maximum "Excellent" rating than to the "Good" rating. However, with the unbalanced 7-label opinion set, the opinion representation would be:

- Value $=$ Excellent

- Location $=$ Excellent

- Sleep Quality = Excellent

- Rooms $=$ Good

- Cleanliness = Good 


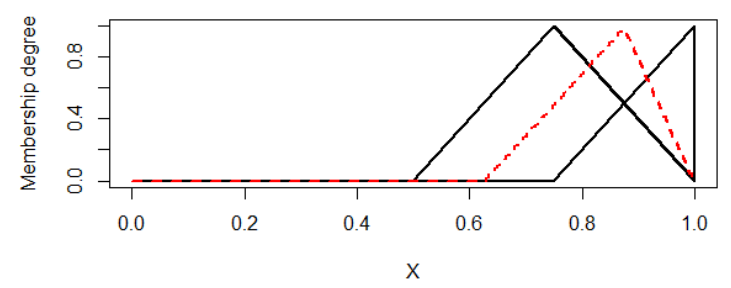

Figure 24: Aggregation with balanced 5-label opinion set

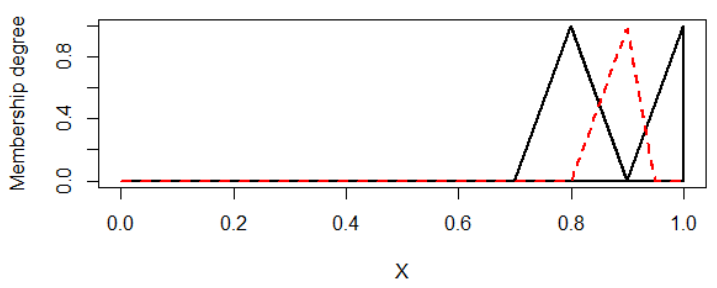

Figure 25: Aggregation with unbalanced 7-label opinion set

- Service $=$ Good

The overall T1OWA outputs is shown in Fig. 25, whose and consequently, with centroid 0.883 more similar to the "Very good" rating than to the maximum "Excellent" rating, which is consistent with the set of individual features rating and therefore would result in a more accurate information on this hotel quality.

\section{Discussion}

Unlike classical approaches for searching opinions, this article presents a more flexible way to characterize an opinion through the different features that describe it. This mechanism allows retrieving opinions taking into account the overall rating and also the individual features, which can have different degrees of importance to represent the needs of a specific user more realistically. Therefore, it is possible to personalize the search of opinions. The success of this proposal is based on the definition of two fuzzy linguistic schemes to represent user's expression of opinions on individual features while, at the same time, allowing them also to express their linguistic preferences on the individual features. The case study proposed shows how flexible this approach can be. Depending on the scheme selected, which can represent different type of users, the aggregation step can result on different outputs reflecting the type of user. Thus, an opinion may be suitable for some users but not for others.

Possible applications of the methodology proposed have been illustrated with examples using real opinions from TripAdvisor. Particular attention has been paid to the use of a balance 5-label opinion set and an unbalanced 7-label opinion set and the difference on their results, which could be further exploited by their embedding into other applications like recommender systems to be able to filter the most suitable products/services regarding a user profile. Another potential application of the aggregation process described to compute the overall rating would be on the classification of users by comparing their individual features ratings with their overall rating of the product/service per se. Some possible applications of this may be, for example, the implementation of quality filters to remove those opinions where the above difference is 'too' large and therefore the information could be seen as inconsistent or misleading. This could be applicable to users, i.e. users with a significant amount of misleading entries/opinions could be banned automatically as they may be considered fake users.

In future, in addition to explicit ratings, the extraction of information from textual reviews/opinions to detect features chosen by the online platform and additional ones to complement explicit ratings would be an interesting research area [50]. Finally, other information different to the different features ratings could also be used in the aggregation step as it is proposed in [51]. 


\section{Conclusions}

A fuzzy approach based on the T1OWA operator to directly fuse individual feature sentiments according to different user profiles has been presented. This methodology overcomes some of the classic systems' issues: the definition of sentiments grounded on numerical values and the inability to search opinions adapted to the users' needs.

Unlike other systems, which allow users to rate a product/service, this approach computes the overall rating automatically based on the individual ratings of the features characterizing such product/service. The success of this proposal is also based on the capability of including different schemes to represent different profiles representing the interests of users or the service providers. The inclusion of an aggregation step based on the T1OWA operator makes the proposal more flexible and adaptable to the needs of the different users.

The examples included and commented show many other opportunities to apply this promising proposal. For example, it may allow detecting fake or incoherent users by designing a filtering of misleading opinions, while it would allow search engines or recommender systems to be designed in a way that they can propose an individual list of products or services according to sentiments and users' profiles.

\section{Acknowledgments}

This work has been partially supported by FEDER and the State Research Agency (AEI) of the Spanish Ministry of Economy and Competition under grant TIN2016-76843-C4-2-R (AEI/FEDER, UE).

\section{References}

[1] Y. Wang, M. Wang, H. Fujita, Word Sense Disambiguation: A comprehensive knowledge exploitation framework, Knowledge-Based Systems In Press.

[2] F. Steiner-Correa, M. I. Viedma-del Jesus, A. G. Lopez-Herrera, A survey of multilingual human-tagged short message datasets for sentiment analysis tasks, Soft Computing 22 (24) (2018) 8227-8242.

[3] O. Araque, G. Zhu, C. A. Iglesias, A semantic similarity-based perspective of affect lexicons for sentiment analysis, Knowledge-Based Systems 165 (2019) 346-359.

[4] R. Moraes, F. Valiati, J, W. P. Gavião Neto, Document-level sentiment classification: An empirical comparison between SVM and ANN, Expert Systems with Applications 40 (2) (2013) 621-633.

[5] A. Duric, F. Song, Feature selection for sentiment analysis based on content and syntax models, Decision Support Systems 53 (4) (2012) 704-711.

[6] Y. He, D. Zhou, Self-training from labeled features for sentiment analysis, Information Processing and Management 47 (4) (2011) 606-616.

[7] F. Ali, D. Kwak, P. Khan, S. El-Sappagh, A. Ali, S. Ullah, K.-H. Kim, K.-S. Kwak, Transportation sentiment analysis using word embedding and ontology-based topic modeling, Knowledge-Based Systems.

[8] G. Li, F. Liu, Application of a clustering method on sentiment analysis, Journal of Information Science 38 (2) (2012) 127-139. 
[9] A. Montoro, J. A. Olivas, A. Peralta, F. P. Romero, J. Serrano-Guerrero, An ANEW based fuzzy sentiment analysis model, in: IEEE International Conference on Fuzzy Systems, IEEE, 2018, pp. 1256-1262.

[10] N. Majumder, D. Hazarika, A. Gelbukh, E. Cambria, S. Poria, Multimodal sentiment analysis using hierarchical fusion with context modeling, Knowledge-Based Systems 161 (2018) 124-133.

[11] O. Appel, F. Chiclana, J. Carter, H. Fujita, A hybrid approach to the sentiment analysis problem at the sentence level, Knowledge-Based Systems 108 (C) (2016) 110-124.

[12] O. Appel, F. Chiclana, J. Carter, H. Fujita, Cross-ratio uninorms as an effective aggregation mechanism in sentiment analysis, Knowledge-Based Systems 124 (C) (2017) 16-22.

[13] O. Appel, F. Chiclana, J. Carter, H. Fujita, A Consensus Approach to the Sentiment Analysis Problem Driven by Support-Based IOWA Majority, International Journal of Intelligent Systems 32 (9) (2017) 947965.

[14] T. Hayashi, H. Fujita, Word Embeddings-based Sentence-Level Sentiment Analysis considering Word Importance, Acta Polytechnica Hungarica 16 (7) (2019) 7-24.

[15] B. A. Ojokoh, O. Kayode, A feature-opinion extraction approach to opinion mining, Journal of Web Engineering 11 (1) (2012) 51-63.

[16] M. Fares, A. Moufarrej, E. Jreij, J. Tekli, W. Grosky, Unsupervised word-level affect analysis and propagation in a lexical knowledge graph, Knowledge-Based Systems 165 (2019) 432-459.

[17] S. Poria, A. Gelbukh, Aspect extraction for opinion mining with a deep convolutional neural network, Knowledge-Based Systems 108 (2016) 42-49.

[18] C. Wu, F. Wu, S. Wu, Z. Yuan, Y. Huang, A hybrid unsupervised method for aspect term and opinion target extraction, Knowledge-Based Systems 148 (2018) 66-73.

[19] J. Wu, F. Chiclana, H. Fujita, E. Herrera-Viedma, A visual interaction consensus model for social network group decision making with trust propagation, Knowledge-Based Systems 122 (2017) 39-50.

[20] N. Capuano, F. Chiclana, H. Fujita, E. Herrera-Viedma, V. Loia, Fuzzy Group Decision Making With Incomplete Information Guided by Social Influence, IEEE Transactions on Fuzzy Systems 26 (3) (2018) 1704-1718.

[21] A.-B. Eliacik, N. Erdogan, Influential user weighted sentiment analysis on topic based microblogging community, Expert Systems with Applications 92 (2018) 403-418.

[22] J. Bernabé-Moreno, A. Tejeda-Lorente, C. Porcel, H. Fujita, E. Herrera-Viedma, Quantifying the emotional impact of events on locations with social media, Knowledge-Based Systems 146 (2018) 44-57.

[23] K. Ravi, V. Ravi, A survey on opinion mining and sentiment analysis: Tasks, approaches and applications, Knowledge-Based Systems 89 (2015) 14-46.

[24] J. Serrano-Guerrero, J. A. Olivas, F. P. Romero, E. Herrera-Viedma, Sentiment analysis: A review and comparative analysis of web services, Information Sciences 311 (1) (2015) 18-38. 
[25] D. Wang, S. Zhu, T. Li, SumView: A Web-based engine for summarizing product reviews and customer opinions, Expert Systems with Applications 40 (1) (2013) 27-33.

[26] K. Ganesan, C. Zhai, J. Han, Opinosis: a graph-based approach to abstractive summarization of highly redundant opinions, in: Proceedings of the 23rd International Conference on Computational Linguistics (COLING' '10), 2010, pp. 340-348.

[27] K. Ganesan, C. Zhai, E. Viegas, Micropinion generation: An Unsupervised Approach to Generating UltraConcise Summaries of Opinions, in: Proceedings of the 21st international conference on World Wide Web - WWW '12, ACM Press, 2012, pp. 869 - 878.

[28] M. Tsytsarau, T. Palpanas, Survey on mining subjective data on the web, Data Mining and Knowledge Discovery 24 (3) (2011) 478-514.

[29] M. E. Basiri, A. R. Naghsh-Nilchi, N. Ghasem-Aghaee, Sentiment prediction based on dempster-shafer theory of evidence, Mathematical Problems in Engineering 2014 (2014) 1-13.

[30] K. Wang, J. N. Liu, W.-M. Ma, Towards the Detection of Potential Contradictions in Fuzzy Ontology Using a High Level Net Approach Integrated with Uncertainty Inference, in: 2010 IEEE International Conference on Data Mining Workshops, IEEE, 2010, pp. 883-890.

[31] C. Sauper, R. Barzilay, Automatic aggregation by joint modeling of aspects and values, Journal of Artificial Intelligence Research 46 (2013) 89-127.

[32] K. Schouten, F. Frasincar, Survey on Aspect-Level Sentiment Analysis, IEEE Transactions on Knowledge and Data Engineering 28 (3) (2016) 813-830.

[33] J. Serrano-Guerrero, J. A. Olivas, F. Romero, Computing Sentiment Analysis through Aspect-based fuzzy Aggregations, in: Conference of the International Fuzzy Systems Association and the European Society for Fuzzy Logic and Technology (EUSFLAT 2019), Atlantis Press, Prague, Czech, 2019, pp. 452-459.

[34] K. Umamaheswari, V. Priya, Aspect Ranking Based on Author Specific Information Aggregation The Proposed Methodology, Journal of Scientific \& Industrial Research 75 (2016) 534-539.

[35] B. Agarwal, N. Mittal, Sentiment Analysis Using ConceptNet Ontology and Context Information, in: N. Mittal (Ed.), Prominent Feature Extraction for Sentiment Analysis, Springer International Publishing, 2016, pp. 63-75.

[36] H. Liu, P. Singh, ConceptNet - A Practical Commonsense Reasoning Tool-Kit, BT Technology Journal 22 (2004) 211-226.

[37] Y. M. Li, T. Y. Li, Deriving market intelligence from microblogs, Decision Support Systems 55 (1) (2013) 206-217.

[38] Z. Zhang, Weighing Stars: Aggregating Online Product Reviews for Intelligent E-commerce Applications, IEEE Intelligent Systems 23 (5) (2008) 42-49.

[39] M. Tsytsarau, T. Palpanas, Managing Diverse Sentiments at Large Scale, IEEE Transactions on Knowledge and Data Engineering 28 (11) (2016) 3028-3040. 
[40] R. R. Yager, On ordered weighted averaging aggregation operators in multicriteria decision making, IEEE transactions on Systems, Man and Cybernetics 18 (1) (1988) 183-190.

[41] S.-M. Zhou, F. Chiclana, J. M. Garibaldi, On properties of Type-1 OWA operators in aggregating uncertain information for soft decision making, International Journal of Intelligent Systems 25 (6) (2008) 1-19.

[42] S.-M. Zhou, F. Chiclana, R. I. John, J. M. Garibaldi, Type-1 OWA operators for aggregating uncertain information with uncertain weights induced by type-2 linguistic quantifiers, Fuzzy Sets and Systems 159 (24) (2008) 3281-3296.

[43] S. M. Zhou, F. Chiclana, R. I. John, J. M. Garibaldi, AlphAlevel Aggregation: A Practical Approach to Type-1 OWA Operation for Aggregating Uncertain Information with Applications to Breast Cancer Treatments, IEEE Transactions on Communications 23 (10) (2011) 1455-1468.

[44] F. Chiclana, F. Mata, L. G. Pérez, E. Herrera-Viedma, Type-1 OWA Unbalanced Fuzzy Linguistic Aggregation Methodology: Application to Eurobonds Credit Risk Evaluation, International Journal of Intelligent Systems 33 (5) (2017) 1071-1078.

[45] R. R. Yager, OWA aggregation over a continuous interval argument with applications to decision making, IEEE Transactions on Systems, Man, and Cybernetics, Part B: Cybernetics 34 (5) (2004) 1952-1963.

[46] C. Chakraborty, D. Chakraborty, A theoretical development on a fuzzy distance measure for fuzzy numbers, Mathematical and Computer Modelling 43 (3-4) (2006) 254-261.

[47] D. J. Dubois, H. M. Prade, Fuzzy sets and systems : Theory and applications, Academic Press, 1980.

[48] E. Szmidt, J. Kacprzyk, Distances between intuitionistic fuzzy sets, Fuzzy Sets and Systems 114 (3) (2000) $505-518$.

[49] L. Zadeh, Outline of a New Approach to the Analysis of Complex Systems and Decision Processes, IEEE Transactions on Systems, Man and Cybernetics SMC-3 (1) (1973) 28-44.

[50] Y. H. Hu, Y. L. Chen, H. L. Chou, Opinion mining from online hotel reviews A text summarization approach, Information Processing and Management 53 (2) (2017) 436-449.

[51] J. Serrano-Guerrero, A. G. Lopez-Herrera, P. Jimenez, J. A. Olivas, E. Herrera-Viedma, Fuzzy methodology for recommendation based on sentiment analysis and content tools, in: SOMET2018: New Trends in Intelligent Software Methodologies, Tools and Techniques, Vol. 303, 2018, pp. 285-298. 